\title{
A note on the higher-order Frobenius-Euler polynomials and Sheffer sequences
}

\section{Dae San Kim ${ }^{1}$, Taekyun Kim²*, Sang-Hun Lee ${ }^{3}$ and Seog-Hoon Rim ${ }^{4}$}

\section{"Correspondence:}

taekyun64@hotmail.com

${ }^{2}$ Department of Mathematics,

Kwangwoon University, Seoul,

139-701, Republic of Korea

Full list of author information is

available at the end of the article

\section{Abstract}

In this paper, we investigate some properties of polynomials related to Sheffer sequences. Finally, we derive some identities of higher-order Frobenius-Euler polynomials.

\section{Introduction}

Let $\lambda(\neq 1) \in \mathbb{C}$. The higher-order Frobenius-Euler polynomials are defined by the generating function to be

$$
\left(\frac{1-\lambda}{e^{t}-\lambda}\right)^{\alpha} e^{x t}=e^{H^{(\alpha)}}(x \mid \lambda) t=\sum_{n=0}^{\infty} H_{n}^{(\alpha)}(x \mid \lambda) \frac{t^{n}}{n !} \quad(\text { see }[1-17])
$$

with the usual convention about replacing $\left(H^{(\alpha)}(x \mid \lambda)\right)^{n}$ by $H_{n}^{(\alpha)}(x \mid \lambda)$. In the special case, $x=0, H_{n}^{(\alpha)}(0 \mid \lambda)=H_{n}^{(\alpha)}(\lambda)$ are called the $n$th Frobenius-Euler numbers of order $\alpha(\in \mathbb{R})$.

From (1) we have

$$
H_{n}^{(\alpha)}(x \mid \lambda)=\sum_{l=0}^{n}\left(\begin{array}{l}
n \\
l
\end{array}\right) H_{n-l}^{(\alpha)}(\lambda) x^{l}=\sum_{l=0}^{n}\left(\begin{array}{l}
n \\
l
\end{array}\right) H_{n-l}^{(\alpha)}(\lambda) x^{n-l} \quad(\text { see }[6]) .
$$

By (2) we get

$$
\frac{\partial}{\partial x} H_{n}^{(\alpha)}(x \mid \lambda)=n H_{n-1}^{(\alpha)}(x \mid \lambda), \quad H_{n}^{(0)}(x \mid \lambda)=x^{n} \quad \text { for } n \in \mathbb{Z}_{+} .
$$

It is not difficult to show that

$$
H_{n}^{(\alpha)}(x+1 \mid \lambda)-\lambda H_{n}^{(\alpha)}(x \mid \lambda)=(1-\lambda) H_{n}^{(\alpha-1)}(x \mid \lambda) \quad(\text { see }[1-17]) .
$$

Let us define the $\lambda$-difference operator $\Delta_{\lambda}$ as follows:

$$
\triangle_{\lambda} f(x)=f(x+1)-\lambda f(x)
$$

(c) 2013 Kim et al.; licensee Springer. This is an Open Access article distributed under the terms of the Creative Commons Attribution License (http://creativecommons.org/licenses/by/2.0), which permits unrestricted use, distribution, and reproduction in any medium, provided the original work is properly cited. 
From (5) we can derive the following equation:

$$
\begin{aligned}
\triangle_{\lambda}^{n} f(x)=\underbrace{\triangle_{\lambda} \cdots \triangle_{\lambda}}_{n \text {-times }} f(x) & =\sum_{k=0}^{n}\left(\begin{array}{l}
n \\
k
\end{array}\right)(-\lambda)^{n-k} f(x+k) \\
& =\sum_{k=0}^{n}\left(\begin{array}{l}
n \\
k
\end{array}\right)(-\lambda)^{k} f(x+n-k) .
\end{aligned}
$$

As is well known, the Stirling numbers $S(l, n)$ of the second kind are defined by the generating function to be

$$
\left(e^{t}-1\right)^{n}=n ! \sum_{l=0}^{\infty} S(l, n) \frac{t^{l}}{l !} \quad(\text { see }[5,6,11])
$$

and

$$
\left(e^{t}-1\right)^{n}=\sum_{l=0}^{\infty}\left(\sum_{m=0}^{n}\left(\begin{array}{l}
n \\
m
\end{array}\right)(-1)^{n-m} m^{l}\right) \frac{t^{l}}{l !} .
$$

By (7) and (8), we get

$$
S(l, n)=\frac{1}{n !} \sum_{m=0}^{n}\left(\begin{array}{l}
n \\
m
\end{array}\right)(-1)^{n-m} m^{l}=\frac{\triangle^{n} 0^{l}}{n !} \quad(\text { see }[11])
$$

where $\Delta f(x)=f(x+1)-f(x)$.

Now, we consider the $\lambda$-analogue of the Stirling numbers of the second kind as follows:

$$
\left(e^{t}-\lambda\right)^{n}=n ! \sum_{l=0}^{\infty} S(l, n \mid \lambda) \frac{t^{l}}{l !}
$$

and

$$
\left(e^{t}-\lambda\right)^{n}=\sum_{l=0}^{\infty}\left(\sum_{m=0}^{n}\left(\begin{array}{l}
n \\
m
\end{array}\right)(-\lambda)^{n-m} m^{l}\right) \frac{t^{l}}{l !} .
$$

From (10) and (11), we have

$$
S(l, n \mid \lambda)=\frac{1}{n !} \sum_{m=0}^{n}\left(\begin{array}{c}
n \\
m
\end{array}\right)(-\lambda)^{n-m} m^{l}=\frac{1}{n !} \triangle_{\lambda}^{n} 0^{l}
$$

and

$$
S(l, n \mid \lambda)=0 \quad \text { for } n>l \text {. }
$$

From (4) and (5), we have

$$
\triangle_{\lambda} H_{n}^{(\alpha)}(x \mid \lambda)=(1-\lambda) H_{n}^{(\alpha-1)}(x \mid \lambda) .
$$


Let $\mathcal{F}$ be the set of all formal power series in the variable $t$ over $\mathbb{C}$ with

$$
\mathcal{F}=\left\{f(t)=\sum_{n=0}^{\infty} \frac{a_{k}}{k !} t^{k} \mid a_{k} \in \mathbb{C}\right\} .
$$

$\mathbb{P}$ indicates the algebra of polynomials in the variable $x$ over $\mathbb{C}$, and $\mathbb{P}^{*}$ is the vector space of all linear functionals on $\mathbb{P}$ (see $[5,11])$. In [11], $\langle L \mid p(x)\rangle$ denotes the action of the linear functional $L$ on a polynomial $p(x)$, and we remind that the vector space structure on $\mathbb{P}^{*}$ is defined by

$$
\begin{aligned}
& \langle L+M \mid p(x)\rangle=\langle L \mid p(x)\rangle+\langle M \mid p(x)\rangle, \\
& \langle c L \mid p(x)\rangle=c|L| p(x)\rangle,
\end{aligned}
$$

where $c$ is a complex constant.

The formal power series

$$
f(t)=\sum_{k=0}^{\infty} \frac{a_{k}}{k !} t^{k} \in \mathcal{F}
$$

defines a linear functional on $\mathbb{P}$ by setting

$$
\left\langle f(t) \mid x^{n}\right\rangle=a_{n} \quad \text { for all } n \in \mathbb{Z}_{+} \text {(see [11]). }
$$

From (15) and (16), we have

$$
\left\langle t^{k} \mid x^{n}\right\rangle=n ! \delta_{n, k} \quad(\text { see }[5,11]) .
$$

Let $f_{L}(t)=\sum_{k=0}^{\infty} \frac{\left\langle L \mid x^{k}\right\rangle}{k !} t^{k}$. From (17) we have

$$
\left\langle f_{L}(t) \mid x^{n}\right\rangle=\left\langle L \mid x^{n}\right\rangle \quad \text { for all } n \in \mathbf{Z}_{+} .
$$

By (18) we get $L=f_{L}(t)$. It is known in [11] that the map $L \mapsto f_{L}(t)$ is a vector space isomorphism from $\mathbb{P}^{*}$ onto $\mathcal{F}$. Henceforth, $\mathcal{F}$ will denote both the algebra of formal power series in $t$ and the vector space of all linear functionals on $\mathbb{P}$, and so an element $f(t)$ of $\mathcal{F}$ will be thought of as both a formal power series and a linear functional. We will call $\mathcal{F}$ the umbral algebra. The umbral calculus is the study of umbral algebra (see $[5,11])$.

The order $O(f(t))$ of the nonzero power series $f(t)$ is the smallest integer $k$ for which the coefficient of $t^{k}$ does not vanish. A series $f(t)$ has $O(f(t))=1$ is called a delta series and a series $f(t)$ has $O(f(t))=0$ is called an invertible series (see [5,11]). By (16) and (17), we get $\left\langle e^{y t} \mid x^{n}\right\rangle=y^{n}$, and so $\left\langle e^{y t} \mid p(x)\right\rangle=p(y)$ (see $\left.[5,11]\right)$. For $f(t) \in \mathcal{F}$ and $p(x) \in \mathbb{P}$, we have

$$
f(t)=\sum_{k=0}^{\infty} \frac{\left\langle f(t) \mid x^{k}\right\rangle}{k !} t^{k}, \quad p(x)=\sum_{k=0}^{\infty} \frac{\left\langle t^{k} \mid p(x)\right\rangle}{k !} x^{k} .
$$


Let $f_{1}(t), f_{2}(t), \ldots, f_{n}(t) \in \mathcal{F}$. Then we see that

$$
\begin{aligned}
& \left\langle f_{1}(t) f_{2}(t) \cdots f_{n}(t) \mid x^{n}\right\rangle \\
& \quad=\sum_{i_{1}+\cdots+i_{m}=n}\left(\begin{array}{c}
n \\
i_{1}, \ldots, i_{m}
\end{array}\right)\left\langle f_{1}(t) \mid x^{i_{1}}\right\rangle \cdots\left\langle f_{m}(t) \mid x^{i_{m}}\right\rangle,
\end{aligned}
$$

where $\left(\begin{array}{c}n \\ i_{1}, \ldots, i_{m}\end{array}\right)=\frac{n !}{i_{1} ! \cdots i_{m} !}($ see $[5,11])$.

For $f(t), g(t) \in \mathcal{F}$ and $p(x) \in \mathbb{P}$, it is easy to show that

$$
\langle f(t) g(t) \mid p(x)\rangle=\langle f(t) \mid g(t) p(x)\rangle=\langle g(t) \mid f(t) p(x)\rangle \quad \text { (see [11]). }
$$

From (19), we can derive the following equation:

$$
p^{(k)}(0)=\left\langle t^{k} \mid p(x)\right\rangle \quad \text { and } \quad\left\langle 1 \mid p^{(k)}(x)\right\rangle=p^{(k)}(0)
$$

By (22) we get

$$
t^{k} p(x)=p^{(k)}(x)=\frac{d^{k} p(x)}{d x^{k}} \quad(\text { see }[5,11])
$$

Thus, from (23) we have

$$
e^{y t} p(x)=p(x+y) .
$$

Let $S_{n}(x)$ be polynomials in the variable $x$ with degree $n$, and let $f(t)$ be a delta series and $g(t)$ be an invertible series. Then there exists a unique sequence $S_{n}(x)$ of polynomials with $\left\langle g(t) f(t)^{k} \mid S_{n}(x)\right\rangle=n ! \delta_{n, k}(n, k \geq 0)$, where $\delta_{n, k}$ is the Kronecker symbol. The sequence $S_{n}(x)$ is called the Sheffer sequence for $(g(t), f(t))$, which is denoted by $S_{n}(x) \sim(g(t), f(t))$. If $S_{n}(x) \sim(1, f(t))$, then $S_{n}(x)$ is called the associated sequence for $f(t)$. If $S_{n}(x) \sim(g(t), t)$, then $S_{n}(x)$ is called the Appell sequence for $g(t)$ (see $[5,11]$ ). For $p(x) \in \mathbb{P}$, the following equations (25)-(27) are known in $[5,11]$ :

$$
\begin{aligned}
& \left\langle\frac{e^{y t}-1}{t} \mid p(x)\right\rangle=\int_{0}^{y} p(u) d u, \\
& \langle f(t) \mid x p(x)\rangle=\left\langle\partial_{t} f(t) \mid p(x)\right\rangle=\left\langle f^{\prime}(t) \mid p(x)\right\rangle,
\end{aligned}
$$

and

$$
\left\langle e^{y t}-1 \mid p(x)\right\rangle=p(y)-p(0)
$$

For $S_{n}(x) \sim(g(t), f(t))$, we have

$$
\begin{aligned}
& h(t)=\sum_{k=0}^{\infty} \frac{\left\langle h(t) \mid S_{k}(x)\right\rangle}{k !} g(t) f(t)^{k}, \quad h(t) \in \mathcal{F}, \\
& p(x)=\sum_{k=0}^{\infty} \frac{\left\langle g(t) f(t)^{k} \mid p(x)\right\rangle}{k !} S_{k}(x), \quad p(x) \in \mathbb{P},
\end{aligned}
$$




$$
\begin{aligned}
& f(t) S_{n}(x)=n S_{n-1}(x), \quad\langle f(t) \mid p(\alpha x)\rangle=\langle f(\alpha t) \mid p(x)\rangle \\
& \frac{1}{g(\bar{f}(t))} e^{y \bar{f}(t)}=\sum_{k=0}^{\infty} \frac{S_{k}(y)}{k !} t^{k} \quad \text { for all } y \in \mathbb{C}
\end{aligned}
$$

where $\bar{f}(t)$ is the compositional inverse of $f(t)$.

$$
S_{n}(x+y)=\sum_{k=0}^{n}\left(\begin{array}{l}
n \\
k
\end{array}\right) P_{k}(y) S_{n-k}(x)=\sum_{k=0}^{n}\left(\begin{array}{l}
n \\
k
\end{array}\right) P_{k}(x) S_{n-k}(y),
$$

where $P_{k}(y)=g(t) S_{k}(y) \sim(1, f(t))$ (see $\left.[5,11]\right)$.

In contrast to the higher-order Euler polynomials, the more general higher-order Frobenius-Euler polynomials have never been studied in the context of umbral algebra and umbral calculus.

In this paper, we investigate some properties of polynomials related to Sheffer sequences. Finally, we derive some identities of higher-order Frobenius-Euler polynomials.

\section{Associated sequences}

Let $p_{n}(x) \sim(1, f(t))$ and $q_{n}(x) \sim(1, g(t))$. Then, for $n \geq 1$, we note that

$$
q_{n}(x)=x\left(\frac{f(t)}{g(t)}\right)^{n} x^{-1} p_{n}(x) \quad(\text { see }[11])
$$

Let us take $f(t)=e^{a t}-1(a \neq 0)$. Then we see that $f^{\prime}(t)=a e^{a t}, \bar{f}(t)=a^{-1} \log (t+1)$.

From (27), we can derive the associated sequence $p_{n}(x)$ for $f(t)=e^{a t}-1$ as follows:

$$
\begin{aligned}
p_{n}(y) & =\left\langle e^{y t} \mid p_{n}(x)\right\rangle=\left\langle e^{y \bar{f}(t)} \mid x^{n}\right\rangle=\left\langle e^{\frac{y}{a} \log (t+1)} \mid x^{n}\right\rangle \\
& =\left\langle(t+1)^{\frac{y}{a}} \mid x^{n}\right\rangle=\sum_{k=0}^{\infty}\left(\begin{array}{l}
\frac{y}{a} \\
k
\end{array}\right)\left\langle t^{k} \mid x^{n}\right\rangle \\
& =\sum_{k=0}^{n}\left(\begin{array}{c}
\frac{y}{a} \\
k
\end{array}\right) n ! \delta_{n, k}=\left(\begin{array}{c}
\frac{y}{a} \\
n
\end{array}\right) n !=\left(\frac{y}{a}\right)_{n},
\end{aligned}
$$

where $(a)_{n}=a(a-1) \cdots(a-n+1)=\prod_{i=0}^{n-1}(a-i)$.

Therefore, by (34) we obtain, for $n \in \mathbb{Z}_{+}$,

$$
p_{n}(x)=\left(\frac{x}{a}\right)_{n} \sim\left(1, e^{a t}-1\right) .
$$

We get the following:

$$
\begin{aligned}
p_{n+1}(x) & =x\left(f^{\prime}(t)\right)^{-1} p_{n}(x)=a^{-1} x e^{-a t} p_{n}(x) \\
& =\left(\frac{x}{a}\right)\left(\frac{x-a}{a}\right)_{n}=\left(\frac{x}{a}\right) p_{n}(x-a) .
\end{aligned}
$$


From (35), we can derive the equation

$$
\begin{aligned}
p_{n+1}(x) & =\left(\frac{x}{a}\right) p_{n}(x-a)=\left(\frac{x}{a}\right)\left(\frac{x-a}{a}\right) p_{n}(x-2 a) \\
& =\left(\frac{x}{a}\right)\left(\frac{x-a}{a}\right)\left(\frac{x-2 a}{a}\right) p_{n}(x-3 a)=\cdots \\
& =\left(\frac{x}{a}\right)\left(\frac{x}{a}-1\right)\left(\frac{x}{a}-2\right) \cdots\left(\frac{x}{a}-n\right) .
\end{aligned}
$$

By (19) we get

$$
\left(\frac{x}{a}\right)_{n}=\sum_{k=0}^{\infty} \frac{\left\langle t^{k} \mid\left(\frac{x}{a}\right)_{n}\right\rangle}{k !} x^{k}=\sum_{k=0}^{n} \frac{S_{1}(n, k)}{a^{k}} x^{k}
$$

and

$$
\frac{1}{k !}\left\langle t^{k} \mid\left(\frac{x}{a}\right)_{n}\right\rangle=\frac{S_{1}(n, k)}{a^{k}}
$$

where $S_{1}(n, k)$ is the Stirling numbers of the first kind.

Therefore, by (37) and (38), we obtain the following theorem.

Lemma 1 For $n, k \geq 0$, we have

$$
\frac{\left\langle t^{k} \mid\left(\frac{x}{a}\right)_{n}\right\rangle}{k !}=\frac{S_{1}(n, k)}{a^{k}} .
$$

From (31) we note that

$$
\sum_{k=0}^{\infty} \frac{\left(\frac{x}{a}\right)_{k}}{k !} t^{k}=e^{\frac{x}{a} \log (1+t)}=(t+1)^{\frac{x}{a}} .
$$

And by (32) we get

$$
\left(\frac{x+y}{a}\right)_{n}=\sum_{k=0}^{n}\left(\begin{array}{l}
n \\
k
\end{array}\right)\left(\frac{x}{a}\right)_{k}\left(\frac{y}{a}\right)_{n-k} .
$$

As is well known, the $n$th Frobenius-Euler polynomials are defined by the generating function to be

$$
\frac{1-\lambda}{e^{t}-\lambda} e^{x t}=\sum_{n=0}^{\infty} H_{n}(x \mid \lambda) \frac{t^{n}}{n !} .
$$

Thus, by (42) we see that $H_{n}(x \mid \lambda) \sim\left(\frac{e^{t}-\lambda}{1-\lambda}, t\right)$. So, we note that

$$
\frac{e^{t}-\lambda}{1-\lambda} H_{n}(x \mid \lambda) \sim(1, t) .
$$


It is easy to show that $x^{n} \sim(1, t)$ (see Eq. (17)). Thus, from (42) we have

$$
\begin{aligned}
x^{n} & =x\left(\frac{t}{t}\right)^{n} x^{-1}\left(\frac{e^{t}-\lambda}{1-\lambda} H_{n}(x \mid \lambda)\right)=\frac{1}{1-\lambda}\left(e^{t}-\lambda\right) H_{n}(x \mid \lambda) \\
& =\frac{1}{1-\lambda}\left(H_{n}(x+1 \mid \lambda)-\lambda H_{n}(x \mid \lambda)\right) .
\end{aligned}
$$

\section{Frobenius-Euler polynomials of order $\alpha$}

From (1) and (31), we note that

$$
H_{n}^{(\alpha)}(x \mid \lambda) \sim\left(\left(\frac{e^{t}-\lambda}{1-\lambda}\right)^{\alpha}, t\right)
$$

and

$$
\left(\frac{1-\lambda}{e^{t}-\lambda}\right)^{\alpha} x^{n}=H_{n}^{(\alpha)}(x \mid \lambda) \quad \text { for all } n \geq 0
$$

From (32), we have

$$
\begin{aligned}
H_{n}^{(\alpha)}(x+y) & =\sum_{k=0}^{n}\left(\begin{array}{l}
n \\
k
\end{array}\right) H_{k}^{(\alpha)}(y \mid \lambda) x^{n-k} \\
& =\sum_{k=0}^{n}\left(\begin{array}{l}
n \\
k
\end{array}\right) H_{k}^{(\alpha)}(x \mid \lambda) y^{n-k} .
\end{aligned}
$$

Let us take the operator $\left(\frac{1-\lambda}{e^{t}-\lambda}\right)^{\beta}$ on both sides of (46).

Then we have

$$
\begin{aligned}
\left(\frac{1-\lambda}{e^{t}-\lambda}\right)^{\beta} H_{n}^{(\alpha)}(x+y \mid \lambda) & =\left(\frac{1-\lambda}{e^{t}-\lambda}\right)^{\beta}\left(\frac{1-\lambda}{e^{t}-\lambda}\right)^{\alpha}(x+y)^{n} \\
& =\left(\frac{1-\lambda}{e^{t}-\lambda}\right)^{\alpha+\beta}(x+y)^{n}=H_{n}^{(\alpha+\beta)}(x+y \mid \lambda)
\end{aligned}
$$

and by (46) we get

$$
\begin{aligned}
H_{n}^{(\alpha+\beta)}(x+y \mid \lambda) & =\sum_{k=0}^{n}\left(\begin{array}{l}
n \\
k
\end{array}\right) H_{k}^{(\alpha)}(y \mid \lambda)\left(\frac{1-\lambda}{e^{t}-\lambda}\right)^{\beta} x^{n-k} \\
& =\sum_{k=0}^{n}\left(\begin{array}{l}
n \\
k
\end{array}\right) H_{k}^{(\alpha)}(y \mid \lambda) H_{n-k}^{(\beta)}(x \mid \lambda) .
\end{aligned}
$$

Therefore, by (48) we obtain the following proposition.

Proposition 2 For $\alpha, \beta \in \mathbb{C}$ and $n \geq 0$, we have

$$
\begin{aligned}
H_{n}^{(\alpha+\beta)}(x+y \mid \lambda) & =\sum_{k=0}^{n}\left(\begin{array}{l}
n \\
k
\end{array}\right) H_{k}^{(\alpha)}(x \mid \lambda) H_{n-k}^{(\beta)}(y \mid \lambda) \\
& =\sum_{k=0}^{n}\left(\begin{array}{l}
n \\
k
\end{array}\right) H_{k}^{(\alpha)}(y \mid \lambda) H_{n-k}^{(\beta)}(x \mid \lambda) .
\end{aligned}
$$


Thus, we have

$$
e^{x t}=\sum_{n=0}^{\infty} H_{n}^{(0)}(x \mid \lambda) \frac{t^{n}}{n !} .
$$

Thus, by (49) we get

$$
H_{n}^{(0)}(x \mid \lambda)=x^{n}
$$

Let us take $\beta=-\alpha$. Then, from Proposition 2, we have

$$
\begin{aligned}
(x+y)^{n} & =\sum_{k=0}^{n}\left(\begin{array}{l}
n \\
k
\end{array}\right) H_{n-k}^{(\alpha)}(x \mid \lambda) H_{k}^{(-\alpha)}(y \mid \lambda) \\
& =\sum_{k=0}^{n}\left(\begin{array}{l}
n \\
k
\end{array}\right) H_{n-k}^{(\alpha)}(y \mid \lambda) H_{k}^{(-\alpha)}(x \mid \lambda) .
\end{aligned}
$$

Therefore, by (51) we obtain the following corollary.

Corollary 3 For $n \geq 0$, we have

$$
\begin{aligned}
(x+y)^{n} & =\sum_{k=0}^{n}\left(\begin{array}{l}
n \\
k
\end{array}\right) H_{n-k}^{(\alpha)}(x \mid \lambda) H_{k}^{(-\alpha)}(y \mid \lambda) \\
& =\sum_{k=0}^{n}\left(\begin{array}{l}
n \\
k
\end{array}\right) H_{n-k}^{(\alpha)}(y \mid \lambda) H_{k}^{(-\alpha)}(x \mid \lambda) .
\end{aligned}
$$

In the special case, $y=0$, we have

$$
x^{n}=\sum_{k=0}^{n}\left(\begin{array}{l}
n \\
k
\end{array}\right) H_{n-k}^{(\alpha)}(x \mid \lambda) H_{k}^{(-\alpha)}(\lambda) .
$$

Let $\alpha \in \mathbb{N}$. We get

$$
\begin{aligned}
\sum_{n=0}^{\infty} \frac{H_{n}^{(-\alpha)}(\lambda)}{n !} t^{n} & =\left(\frac{e^{t}-\lambda}{1-\lambda}\right)^{\alpha} \\
& =\frac{1}{(1-\lambda)^{\alpha}} \sum_{l=0}^{\alpha}\left(\begin{array}{l}
\alpha \\
l
\end{array}\right)(-1)^{\alpha-l} \lambda^{\alpha-l} e^{l t} \\
& =\sum_{n=0}^{\infty}\left(\frac{1}{(1-\lambda)^{\alpha}} \sum_{l=0}^{\alpha}\left(\begin{array}{l}
\alpha \\
l
\end{array}\right)(-1)^{\alpha-l} \lambda^{\alpha-l} l^{n}\right) \frac{t^{n}}{n !}
\end{aligned}
$$

Thus, from (52) we have

$$
\begin{aligned}
H_{n}^{(-\alpha)}(\lambda) & =\frac{1}{(1-\lambda)^{\alpha}} \sum_{l=0}^{\alpha}\left(\begin{array}{l}
\alpha \\
l
\end{array}\right)(-1)^{\alpha-l} \lambda^{\alpha-l} l^{n} \\
& =\frac{1}{(1-\lambda)^{\alpha}} \triangle_{\lambda}^{\alpha} 0^{n}=\frac{\alpha !}{(1-\lambda)^{\alpha}} \frac{\triangle_{\lambda}^{\alpha} 0^{n}}{\alpha !}=\frac{\alpha !}{(1-\lambda)^{\alpha}} S(n, \alpha \mid \lambda) .
\end{aligned}
$$

Therefore, by (51), (52) and (53), we obtain the following theorem. 
Theorem 4 For $\alpha \in \mathbb{N}$ and $n \geq 0$, we have

$$
\frac{(1-\lambda)^{\alpha}}{\alpha !} x^{n}=\sum_{k=0}^{n}\left(\begin{array}{l}
n \\
k
\end{array}\right) H_{n-k}^{(\alpha)}(x \mid \lambda) S(k, \alpha \mid \lambda) .
$$

From (19), we have

$$
\begin{aligned}
x^{n} & =\sum_{k=0}^{n} \frac{\left\langle\left(\frac{e^{t}-\lambda}{1-\lambda}\right)^{\alpha} t^{k} \mid x^{n}\right\rangle}{k !} H_{k}^{(\alpha)}(x \mid \lambda) \\
& =\sum_{k=0}^{n} \frac{1}{k !}\left\langle\left(\frac{e^{t}-\lambda}{1-\lambda}\right)^{\alpha} \mid t^{k} x^{n}\right\rangle H_{k}^{(\alpha)}(x \mid \lambda) \\
& =\sum_{k=0}^{n}\left(\begin{array}{l}
n \\
k
\end{array}\right)\left\langle\left(\frac{e^{t}-\lambda}{1-\lambda}\right)^{\alpha} \mid x^{n-k}\right\rangle H_{k}^{(\alpha)}(x \mid \lambda)
\end{aligned}
$$

and

$$
\begin{aligned}
\left\langle\left(\frac{e^{t}-\lambda}{1-\lambda}\right)^{\alpha} \mid x^{n-k}\right\rangle & =\sum_{j=0}^{\infty} \frac{H_{j}^{(-\alpha)}(\lambda)}{j !}\left\langle t^{j} \mid x^{n-k}\right\rangle \\
& =\sum_{j=0}^{\infty} \frac{H_{j}^{(-\alpha)}(\lambda)}{j !} \delta_{n-k, j}(n-k) ! \\
& =H_{n-k}^{(-\alpha)}(\lambda) .
\end{aligned}
$$

By (54) and (55), we also get

$$
x^{n}=\sum_{k=0}^{n}\left(\begin{array}{l}
n \\
k
\end{array}\right) H_{k}^{(\alpha)}(x \mid \lambda) H_{n-k}^{(-\alpha)}(\lambda) .
$$

\section{Further remark}

Let us take $a=1$ in (34). Then we have $(x)_{n} \sim\left(1, f(t)=e^{t}-1\right), x^{n} \sim(1, g(t)=t)$.

For $n \geq 1$, by (33) we get

$$
\begin{aligned}
x^{n} & =x\left(\frac{e^{t}-1}{t}\right)^{n} x^{-1}(x)_{n}=x\left(\frac{e^{t}-1}{t}\right)^{n}(x-1)_{n-1} \\
& =x \sum_{l=0}^{\infty} \frac{n !}{(l+n) !} S_{2}(l+n, n) t^{l}(x-1)_{n-1},
\end{aligned}
$$

where $S_{2}(m, n)$ is the Stirling numbers of the second kind.

From (56) we have

$$
(x+1)^{n+1}=(x+1) \sum_{l=0}^{n} \frac{(n+1) !}{(l+n+1) !} S_{2}(l+n+1, n+1) t^{l}(x)_{n},
$$

where

$$
(x)_{n}=\sum_{l=0}^{n} S_{1}(n, k) x^{k}
$$


Thus, by (58) we get

$$
t^{l}(x)_{n}=\frac{d^{l}}{d t^{l}}(x)_{n}=\sum_{k=l}^{n} S_{1}(n, k)(k)_{l} x^{k-l}
$$

From (57) and (59), we can derive the following equation:

$$
\begin{aligned}
(x+1)^{n} & =\sum_{l=0}^{n} \frac{(n+1) !}{(l+n+1) !} S_{2}(l+n+1, n+1) \sum_{k=l}^{n} S_{1}(n, k)(k) x_{l} x^{k-l} \\
& =\sum_{l=0}^{n} \sum_{m=0}^{n-l} \frac{\left(\begin{array}{c}
l+m \\
l
\end{array}\right)}{\left(\begin{array}{c}
n+l+1 \\
l
\end{array}\right)} S_{2}(l+n+1, n+1) S_{1}(n, l+m) x^{m} \\
& =\sum_{m=0}^{n}\left\{\sum_{l=0}^{n-m} \frac{\left(\begin{array}{c}
l+m \\
l
\end{array}\right)}{\left(\begin{array}{c}
n+l+1 \\
l
\end{array}\right)} S_{2}(l+n+1, n+1) S_{1}(n, l+m)\right\} x^{m}
\end{aligned}
$$

and

$$
(x+1)^{n}=\sum_{l=0}^{n}\left(\begin{array}{l}
n \\
l
\end{array}\right) x^{l}
$$

Therefore, by (60) and (61), we obtain the following lemma.

Lemma 5 For $0 \leq m \leq n$, we have

$$
\left(\begin{array}{l}
n \\
m
\end{array}\right)=\sum_{l=0}^{n-m} \frac{\left(\begin{array}{c}
l+m \\
l
\end{array}\right)}{\left(\begin{array}{c}
n+l+1 \\
l
\end{array}\right)} S_{2}(l+n+1, n+1) S_{1}(n, l+m) .
$$

Let $\alpha=1$. Then we write $H_{n}^{(1)}(x \mid \lambda)=H_{n}(x \mid \lambda)$. From (34), we note that

$$
(x)_{n} \sim\left(1, e^{t}-1\right)
$$

Thus, by (19) and (62), we get

$$
\begin{aligned}
H_{n}(x \mid \lambda) & =\sum_{k=0}^{\infty} \frac{\left\langle\left(e^{t}-1\right)^{k} \mid H_{n}(x \mid \lambda)\right\rangle}{k !}(x)_{k} \\
& =H_{n}(\lambda)+\sum_{k=1}^{\infty} \frac{\left\langle\left(e^{t}-1\right)^{k} \mid H_{n}(x \mid \lambda)\right\rangle}{k !}(x)_{k} .
\end{aligned}
$$

For $k \geq 1$, we have

$$
\left\langle\left(e^{t}-1\right)^{k} \mid H_{n}(x \mid \lambda)\right\rangle=\sum_{l=0}^{n}\left(\begin{array}{l}
n \\
l
\end{array}\right) H_{n-l}(\lambda)\left\langle\left(e^{t}-1\right)^{k} \mid x^{l}\right\rangle .
$$

From (9), we have

$$
\frac{1}{k !}\left\langle\left(e^{t}-1\right)^{k} \mid x^{l}\right\rangle=S_{2}(l, k) .
$$

Therefore, by (63), (64) and (65), we obtain the following theorem. 
Theorem 6 For $n \geq 0$, we have

$$
\begin{aligned}
H_{n}(x \mid \lambda) & =H_{n}(\lambda)+\sum_{k=1}^{n} \sum_{l=0}^{n}\left(\begin{array}{l}
n \\
l
\end{array}\right) H_{n-l}(\lambda) S_{2}(l, k)(x)_{k} \\
& =H_{n}(\lambda)+\sum_{k=1}^{n} \sum_{l=0}^{n} \sum_{m=0}^{k}\left(\begin{array}{l}
n \\
l
\end{array}\right) H_{n-l}(\lambda) S_{2}(l, k) S_{1}(k, m) x^{m} .
\end{aligned}
$$

From the recurrence formula of the Appell sequence, we note that

$$
\begin{aligned}
H_{n+1}^{(\alpha)}(x \mid \lambda) & =\left(x-\alpha \frac{e^{t}}{e^{t}-\lambda}\right) H_{n}^{(\alpha)}(x \mid \lambda)=x H_{n}^{(\alpha)}(x \mid \lambda)-\frac{\alpha e^{t}}{e^{t}-\lambda} H_{n}^{(\alpha)}(x \mid \lambda) \\
& =x H_{n}^{(\alpha)}(x \mid \lambda)-\frac{\alpha}{(1-\lambda)} \frac{1-\lambda}{e^{t}-\lambda} H_{n}^{(\alpha)}(x+1) \\
& =x H_{n}^{(\alpha)}(x \mid \lambda)-\frac{\alpha}{(1-\lambda)} H_{n}^{(\alpha+1)}(x+1) .
\end{aligned}
$$

Therefore, by (66) we obtain the following theorem.

Theorem 7 For $n \geq 0$, we have

$$
H_{n+1}^{(\alpha)}(x \mid \lambda)=x H_{n}^{(\alpha)}(x \mid \lambda)-\frac{\alpha}{(1-\lambda)} H_{n}^{(\alpha+1)}(x+1) .
$$

\section{Competing interests}

The authors declare that they have no competing interests.

\section{Authors' contributions}

All authors contributed equally to the manuscript and typed, read, and approved the final manuscript.

\section{Author details}

'Department of Mathematics, Sogang University, Seoul, 121-742, Republic of Korea. ${ }^{2}$ Department of Mathematics, Kwangwoon University, Seoul, 139-701, Republic of Korea. ${ }^{3}$ Division of General Education, Kwangwoon University, Seoul, 139-701, Republic of Korea. ${ }^{4}$ Department of Mathematics Education, Kyungpook National University, Taegu, 702-701, Republic of Korea.

\section{Acknowledgements}

Dedicated to Professor Hari M Srivastava.

The authors express their sincere gratitude to the referees for their valuable suggestions and comments. This paper is supported in part by the Research Grant of Kwangwoon University in 2013.

Received: 9 December 2012 Accepted: 6 February 2013 Published: 21 February 2013

\section{References}

1. Araci, S, Acikgoz, M: A note on the Frobenius-Euler numbers and polynomials associated with Bernstein polynomials. Adv. Stud. Contemp. Math. 22, 399-406 (2012)

2. Can, M, Cenkci, M, Kurt, V, Simsek, Y: Twisted Dedekind type sums associated with Barnes' type multiple Frobenius-Euler I-functions. Adv. Stud. Contemp. Math. 18, 135-160 (2009)

3. Carlitz, L: The product of two Eulerian polynomials. Math. Mag. 36, 37-41 (1963)

4. Kim, T: Identities involving Frobenius-Euler polynomials arising from non-linear differential equations. J. Number Theory 132(12), 2854-2865 (2012)

5. Kim, DS, Kim, T: Some new identities of Frobenius-Euler numbers and polynomials. J. Inequal. Appl. 2012, 307 (2012)

6. Kim, T: An identity of the symmetry for the Frobenius-Euler polynomials associated with the fermionic $p$-adic invariant $q$-integrals on $Z_{p}$. Rocky Mt. J. Math. 41, 239-247 (2011)

7. Kim, T: On explicit formulas of $p$-adic q-L-functions. Kyushu J. Math. 48, $73-86$ (1994)

8. Kim, T, Choi, J: A note on the product of Frobenius-Euler polynomials arising from the $p$-adic integral on $Z_{p}$. Adv. Stud. Contemp. Math. 22, 215-223 (2012)

9. Kim, T: A note on q-Bernstein polynomials. Russ. J. Math. Phys. 18(1), 73-82 (2011)

10. Kim, DS, Kim, T, Kim, YH, Dolgy, DV: A note on Eulerian polynomials associated with Bernoulli and Euler numbers and polynomials. Adv. Stud. Contemp. Math. 22(3), 379-389 (2012) 
11. Roman, S: The Umbral Calculus. Dover, New York (2005)

12. Kim, DS, Kim, T, Lee, S-H, Rim, S-H: Frobenius-Euler polynomials and umbral calculus in the $p$-adic case. Adv. Differ. Equ. 2012, 222 (2012)

13. Kim, DS, Kim, T: Euler basis, identities, and their applications. Int. J. Math. Math. Sci. 2012, Article ID 343981 (2012)

14. Rim, S-H, Joung, J, Jin, J-H, Lee, S-J: A note on the weighted Carlitz's type $q$-Euler numbers and $q$-Bernstein polynomials. Proc. Jangjeon Math. Soc. 15, 195-201 (2012)

15. Ryoo, CS: A note on the Frobenius-Euler polynomials. Proc. Jangjeon Math. Soc. 14, 495-501 (2011)

16. Simsek, Y, Yurekli, O, Kurt, V: On interpolation functions of the twisted generalized Frobenius-Euler numbers. Adv. Stud. Contemp. Math. 15, 187-194 (2007)

17. Shiratani, K, Yamamoto, S: On a p-adic interpolation function for the Euler numbers and its derivatives. Mem. Fac. Sci., Kyushu Univ., Ser. A, Math. 39, 113-125 (1985)

doi:10.1186/1687-1847-2013-41

Cite this article as: Kim et al.: A note on the higher-order Frobenius-Euler polynomials and Sheffer sequences. Advances in Difference Equations 2013 2013:41.

Submit your manuscript to a SpringerOpen ${ }^{\odot}$ journal and benefit from:

- Convenient online submission

Rigorous peer review

- Immediate publication on acceptance

- Open access: articles freely available online

- High visibility within the field

- Retaining the copyright to your article 